\title{
FATORES PERINATAIS ASSOCIADOS AO DESCONFORTO RESPIRATÓRIO DO RECÉM-NASCIDO
}

\section{PERINATAL FACTORS ASSOCIATED WITH RESPIRATORY DISCOMFORT IN THE NEWBORN \\ FACTORES PERINATALES ASOCIADOS CON MALESTAR RESPIRATORIO EN EL RECIÉN NACIDO}

Fabiane Blanco Silva Bernardino ${ }^{1}$, Danielly Silva Rodrigues², Millena Mikaella Sousa Santos ${ }^{2}$, Mariana Camargo Tanaka ${ }^{3}$, Bruna Hinnah Borges Martins de Freitas ${ }^{4}$, Maria Aparecida Munhoz Gaíva ${ }^{5}$

\section{RESUMO}

Objetivo: Analisar a prevalência e fatores perinatais associados ao desconforto respiratório em neonatos internados em uma Unidade de Terapia Intensiva Neonatal em Cuiabá, Mato Grosso. Método: Estudo transversal, analítico, retrospectivo, com coleta dos dados entre os meses de outubro e dezembro de 2019, em 844 prontuários de recém-nascidos internados entre 2014 e 2018. Utilizou-se a Regressão de Poisson, considerando significância de 0,05 ( $p<0,05)$. Resultados: do total de neonatos, 49,05\% foram diagnosticados com desconforto respiratório. A prevalência da doença foi $60 \%$ maior entre os pré-termos, $36 \%$ maior entre os que apresentaram histórico de uso materno de esteroide antenatal e $25 \%$ maior entre os de baixo peso ao nascer. Observou-se, ainda, que a prevalência do uso de capacete de oxigênio, pressão positiva contínua nas vias aéreas e dieta parenteral foi $91 \%$, $89 \%$ e $18 \%$ maior entre os neonatos com a doença. No entanto, o uso de fórmulas e leite materno em neonatos com desconforto respiratório foi $85 \%$ e $62 \%$ menor do que os neonatos que não apresentavam tal condição. Conclusão: Indica-se maior atenção da equipe de saúde e gestores, uma vez que o conhecimento desses fatores poderá auxiliá-los no planejamento de ações para consolidação da rede de atenção perinatal, com reestruturação e qualificação dos processos assistenciais no pré-natal, parto e nascimento.

Descritores: Unidade de Terapia Intensiva Neonatal; Assistência Perinatal; Síndrome do Desconforto Respiratório do Recém-nascido; Morbidade; Enfermagem.

\section{ABSTRACT}

Objective: To analyze the prevalence of perinatal factors associated with respiratory distress in neonates admitted to a Neonatal Intensive Care Unit in Cuiabá, Mato Grosso. Method: A cross-sectional, analytical, retrospective study, with data collection between the months of October and December 2019, in 844 medical records of newborns hospitalized between 2014 and 2018 . Poisson Regression was used, considering a significance of 0.05 ( $p<0.05$ ). Results: Of the total number of neonates, $49.05 \%$ were diagnosed with respiratory distress. The prevalence of the disease was $60 \%$ higher among premature infants, $36 \%$ higher among those who had a history of maternal use of antenatal steroids, and $25 \%$ higher among those with low weight. It was also observed that the prevalence of use of an oxygen helmet, the continuous positive airway pressure and the parenteral diet was $91 \%, 89 \%$ and $18 \%$ higher among neonates with the disease, respectively. However, the use of formulas and breast milk in infants with discomfort was $85 \%$ and $62 \%$ lower than that of infants who did not have this condition. Conclusion: Greater attention by the health team and managers is necessary as knowledge of these factors may assist them in planning actions to consolidate the perinatal network, with restructuring and qualification of care processes in prenatal aid, delivery and birth.

Descriptors: Intensive Care Units, Neonatal; Perinatal Care; Respiratory Distress Syndrome, Newborn; Morbidity; Nursing.

\section{RESUMEN}

Objetivo: Analizar el predominio y los factores perinatales asociados a la dificultad respiratoria en neonatos ingresados en una Unidad de Cuidados Intensivos Neonatales de Cuiabá, Mato Grosso. Método: Estudio transversal, analítico y retrospectivo, con recolección de datos entre los meses de octubre a diciembre de 2019, en 844 historias clínicas de recién nacidos hospitalizados entre 2014 y 2018. Se utilizó Regresión de Poisson, considerando una significancia de 0.05 ( $p<0,05)$. Resultados: Del total de neonatos, el $49,05 \%$ fueron diagnosticados de dificultad respiratoria. El predominio de la enfermedad fue un $60 \%$ más alto entre los bebés prematuros, un $36 \%$ más alto entre los que tenían antecedentes de uso materno de esteroides prenatales y un $25 \%$ más alto entre los que tenían bajo peso. También se observó que el predominio del casco de oxígeno, presión positiva continua en las vías respiratorias y dieta parenteral fue $91 \%, 89 \%$ y $18 \%$ mayor entre los recién nacidos con la enfermedad. Sin embargo, el uso de fórmulas y leche materna en lactantes con malestar fue $85 \%$ y $62 \%$ menor que el de lactantes que no padecían esta afección. Conclusión: Se indica una mayor atención por parte del equipo de salud y los gestores, ya que el conocimiento de estos factores puede ayudarlos a planificar acciones para consolidar la red perinatal, con reestructuración y calificación de los procesos de atención en el prenatal, parto y nacimiento. Descriptores: Unidades de Cuidado Intensivo Neonatal; Atención Perinatal; Síndrome de Dificultad Respiratoria del Recién Nacido; Morbilidad; Enfermería.

${ }^{1}$ Doutora em Ciências. Docente da Faculdade de Enfermagem e do Programa de Pós-graduação em Enfermagem da Universidade Federal de Mato Grosso. ${ }^{2}$ Acadêmicas da Faculdade de Enfermagem da Universidade Federal de Mato Grosso. ${ }^{3}$ Mestranda do Programa de Pós-graduação em Enfermagem da Universidade Federal de Mato Grosso. ${ }^{4}$ Mestra em Enfermagem. Docente da Faculdade de Enfermagem da Universidade Federal de Mato Grosso. ${ }^{5}$ Doutora em Ciências. Pesquisadora Associada do Programa de Pós-graduação em Enfermagem da Universidade Federal de Mato Grosso.

\section{Como citar este artigo:}

Bernardino FBS, Rodrigues DS, Santos MMK, et al. Fatores perinatais associados ao desconforto respiratório do recém-nascido. Revista de Enfermagem do Centro-Oeste Mineiro. 2020;10:e3960. [Access___]; Available in:___. DOI: http://doi.org/10.19175/recom.v10i0.3960 


\section{INTRODUÇÃO}

O Desconforto Respiratório do Recémnascido (DRRN) configura-se como um dos agravos mais comuns entre os neonatos e se subdivide em síndrome do desconforto respiratório e a taquipneia transitória do recém-nascido ${ }^{(1)}$.

Os neonatos acometidos pelo DRRN apresentam como principais sintomas batimento das aletas nasais, retrações torácicas, taquipneia, apneia, gemência expiatória e cianose central e de extremidade. A sua etiologia está relacionada à imaturidade dos alvéolos pulmonares, diminuição na produção de surfactante endógeno, baixa maturidade da musculatura pulmonar e retardo na remoção de líquido pulmonar fetal(1-2), ligados à condição do neonato intraútero e em resposta ao nascimento, por isso, o período perinatal, que abrange, desde a 20 semana gestacional até o $28^{\circ}$ dia, após o nascimento, assume importância e relação com o aparecimento da doença.

Estudos evidenciam associação significativa entre fatores perinatais com o DRRN, tais como parto prematuro; parto cesáreo; peso ao nascer menor que 2.500g; uso insuficiente de corticosteroide antenatal por gestantes e de surfactante no período neonatal imediato; índice de Apgar menor que 7; ruptura prematura de membrana e sexo masculino ${ }^{(2-5)}$.

Ademais, os RN com DRRN apresentam também necessidade, significativamente, maior de reanimação, intubação endotraqueal, oxigenoterapia, ventilação invasiva precoce, nutrição parenteral e hospitalização prolongada na Unidade de Terapia Intensiva Neonatal (UTIN) após o nascimento ${ }^{(3)}$.

Além da estreita associação com os fatores citados, anteriormente, o DRRN aparece como variável importante no agravamento do cenário de morbimortalidade perinatal ${ }^{(5)}$, representando a segunda maior causa de mortalidade neonatal precoce $^{(6)}$ e a principal causa de internação em UTIN no Brasil(7), o qual acarreta gastos e prejuízos à saúde pública ${ }^{(5)}$.

Apesar da relevância desse agravo, poucas investigações foram feitas sobre os fatores perinatais associados a essa afecção. Portanto, a identificação desses fatores poderá subsidiar proposições de medidas preventivas em saúde e identificação de ações que poderão contribuir para a redução da morbimortalidade relacionada a esse agravo. Nesse sentido, o presente estudo objetivou analisar a prevalência e os fatores perinatais associados ao desconforto respiratório em neonatos internados em uma UTIN de Cuiabá, Mato Grosso.

\section{MÉTODO}

Estudo transversal, analítico, retrospectivo, realizado a partir de fonte de dados secundários (prontuários), em um hospital universitário público de Cuiabá, Mato Grosso, o qual possui uma UTIN com 10 leitos e é referência na atenção maternoinfantil, principalmente de gestação de alto risco. Incluíram-se todos os neonatos internados na UTIN do referido hospital, no período de 2014 a 2018. A coleta dos dados ocorreu entre os meses de outubro e novembro de 2019.

A variável de interesse foi o DRRN (sim e não). Os neonatos classificados como "sim" para DRRN foram os diagnosticados de acordo com a $10^{\circ}$ Revisão da Classificação Internacional de Doenças (CID), que o classifica em síndrome da angústia respiratória do recém-nascido (P22.0), taquipneia respiratória do recém-nascido (P22.1), outros desconfortos respiratórios do recémnascido (P22.8) e desconforto respiratório não especificado do recém-nascido (P22.9).

As demais variáveis foram as perinatais e as relacionadas às intervenções clínicas, a saber: sexo (masculino e feminino); parto cesáreo (sim e não); idade gestacional (pré-termo $<37$ semanas, termo de 37 a 42 semanas e pós-termo $\geq 42$ semanas); baixo peso ao nascer ( $<2.500 \mathrm{~g}$ ) (sim e não); Apgar no 10 minuto (baixo $<7$ e adequado $\geq 7$ ); Apgar no 50 minuto (baixo $<7$ e adequado $\geq 7$ ); reanimação ao nascer (sim e não); uso de esteroide antenatal (sim ou não); uso de surfactante (sim e não); uso de suporte ventilatório: capacete de oxigênio (HOOD) (sim e não); Pressão Positiva Contínua nas Vias Aéreas (CPAP) (sim e não) e ventilação mecânica (sim e não); e tipo de nutrição, após o nascimento (leite materno (LM), leite humano pasteurizado (LHP), fórmula e nutrição parenteral).

A estruturação do banco de dados foi realizada, por meio do editor de planilha da Microsoft Office Excel ${ }^{\circledR}$ com dupla digitação independente. Os dados de ambos os bancos foram comparados, utilizando a ferramenta Data Compare para identificar possíveis incoerências na digitação, posteriormente, corrigidas pela consulta aos documentos de coleta de dados físicos.

Utilizaram-se cálculos de frequência absoluta e relativa na análise descritiva. Calculouse a Razão de Prevalência, por meio da Regressão 
de Poisson com variância robusta, para comparar a prevalência entre as variáveis, considerando um nível de significância de 0,05 $(p<0,05)$. A análise dos dados foi realizada pelo software $\operatorname{STATA}^{\circledR}$, versão 11.1.

A pesquisa foi aprovada pelo Comitê de Ética em Pesquisa, obtendo parecer consubstanciado no 2.788.928, e considerando as características da pesquisa foi solicitada a dispensa do Termo de Consentimento Livre e Esclarecido (TCLE), mas foram respeitadas todas as recomendações da Resolução 466/2012 do Conselho Nacional de Saúde.

\section{RESULTADOS}

Analisaram-se 844 prontuários de RN internados na UTIN, no período de 2014 a 2018, destes 414 (49,05\%) apresentaram o diagnóstico de DRRN. Entre os RN, a maioria era do sexo masculino $(n=462 ; 56,20 \%)$, prematura $(n=533$; $64,92 \%)$, nasceu de parto cesáreo ( $n=596$; $71,46 \%)$, com baixo peso ( $n=468 ; 56,12 \%$ ) e tinha histórico de uso materno de esteroide antenatal ( $n=511 ; 81,37 \%)$.

Também, destaca-se que 237 (29,77\%) neonatos apresentaram Apgar baixo no 10 minuto, 56 (7,04\%) no 5o minuto, 179 (25,35\%) necessitaram de reanimação, 481 (58,66\%) fizeram uso de suporte respiratório com HOOD e 262 (32,59\%) usaram ventilação mecânica. Ainda, a maior parte dos RN não fez uso de surfactante ( $n=610 ; 85,67 \%)$. Quanto à primeira alimentação, identificou-se que a maioria dos $\mathrm{RN}$ recebeu a dieta do tipo parenteral ( $n=444 ; 54,41 \%)$, seguida do leite humano pasteurizado ( $n=250 ; 30,64 \%$ ), conforme a Tabela 1.

Tabela 1 - Caracterização dos recém-nascidos segundo as variáveis perinatais. Cuiabá, Mato Grosso, Brasil, 2019.

\begin{tabular}{|c|c|c|}
\hline Variáveis & $\bar{n}$ & $\%$ \\
\hline \multicolumn{3}{|c|}{ Desconforto respiratório do recém-nascido $(n=844)$} \\
\hline Não & 430 & 50,95 \\
\hline Sim & 414 & 49,05 \\
\hline \multicolumn{3}{|l|}{ Sexo $(n=822)$} \\
\hline Feminino & 360 & 43,80 \\
\hline Masculino & 462 & 56,20 \\
\hline \multicolumn{3}{|c|}{ Parto cesáreo (n=834) } \\
\hline Não & 238 & 28,54 \\
\hline Sim & 596 & 71,46 \\
\hline \multicolumn{3}{|c|}{ Idade gestacional $(n=821)$} \\
\hline Pré-termo & 533 & 64,92 \\
\hline Termo & 284 & 34,59 \\
\hline Pós-termo & 4 & 0,49 \\
\hline \multicolumn{3}{|c|}{ Baixo Peso $(n=834)$} \\
\hline Não & 366 & 43,88 \\
\hline Sim & 468 & 56,12 \\
\hline \multicolumn{3}{|c|}{ Apgar 19 minuto $(n=796)$} \\
\hline Baixo & 237 & 29,77 \\
\hline Adequado & 559 & 70,23 \\
\hline \multicolumn{3}{|c|}{ Apgar 5o minuto $(n=796)$} \\
\hline Baixo & 56 & 7,04 \\
\hline Adequado & 740 & 92,96 \\
\hline \multicolumn{3}{|c|}{ Uso de esteroide antenatal $(n=628)$} \\
\hline Não & 511 & 81,37 \\
\hline Sim & 117 & 18,63 \\
\hline \multicolumn{3}{|c|}{ Reanimação neonatal ( $n=706$ ) } \\
\hline Não & 527 & 74,65 \\
\hline Sim & 179 & 25,35 \\
\hline \multicolumn{3}{|c|}{ Uso de surfactante $(n=712)$} \\
\hline Não & 610 & 85,67 \\
\hline$\leq 2$ horas & 41 & 5,76 \\
\hline 2- 4 horas & 15 & 2,11 \\
\hline$>4$ horas & 46 & 6,46 \\
\hline \multicolumn{3}{|c|}{ Suporte respiratório com HOOD* $(n=820)$} \\
\hline Não & 339 & 41,34 \\
\hline Sim & 481 & 58,66 \\
\hline
\end{tabular}




\begin{tabular}{|c|c|c|}
\hline Variáveis & n & $\%$ \\
\hline \multicolumn{3}{|c|}{ Suporte respiratório com CPAP† ( $n=795)$} \\
\hline Não & 554 & 69,69 \\
\hline Sim & 241 & 30,31 \\
\hline \multicolumn{3}{|l|}{ Ventilação mecânica ( $n=804$ ) } \\
\hline Não & 542 & 67,41 \\
\hline Sim & 262 & 32,59 \\
\hline \multicolumn{3}{|l|}{ 1a Alimentação (n=816) } \\
\hline Leite Materno & 107 & 13,11 \\
\hline Leite Humano Pasteurizado & 250 & 30,64 \\
\hline Fórmula & 15 & 1,84 \\
\hline Parenteral & 444 & 54,41 \\
\hline
\end{tabular}

Fonte: elaborada pelas autoras com base nos dados da pesquisa.

Nota: *Capacete de oxigênio; + Continuous Positive Airway Pressure/ pressão positiva contínua nas vias aéreas.

Constatou-se que a prevalência de DRRN foi $60 \%$ maior entre os neonatos pré-termos, $36 \%$ maior entre os que apresentaram histórico de uso materno de esteroide antenatal e $25 \%$ maior entre os de baixo peso $(p<0,01)$. As demais variáveis perinatais não apresentaram diferença estatisticamente significante conforme pode ser visualizado na Tabela 2.

Tabela 2 - Fatores perinatais associados ao desconforto respiratório do recém-nascido. Cuiabá, Mato Grosso, Brasil, 2019.

\begin{tabular}{|c|c|c|c|c|c|c|}
\hline \multirow{3}{*}{ Variáveis } & \multicolumn{4}{|c|}{ Desconforto respiratório do recém-nascido } & \multicolumn{2}{|c|}{ Regressão de Poisson simples } \\
\hline & \multicolumn{2}{|c|}{ Não } & \multicolumn{2}{|c|}{ Sim } & \multirow[t]{2}{*}{$\mathrm{RP}\left(\mathrm{IC}_{95 \%)}\right.$} & \multirow[t]{2}{*}{ p-valor } \\
\hline & $\mathbf{n}$ & $\% *$ & $\mathbf{n}$ & $\% *$ & & \\
\hline \multicolumn{7}{|l|}{ Sexo } \\
\hline Feminino & 179 & 43,45 & 181 & 44,15 & 1 & \\
\hline Masculino & 233 & 56,55 & 229 & 55,85 & $0,98(0,86-1,13)$ & 0,84 \\
\hline \multicolumn{7}{|l|}{ Parto cesáreo } \\
\hline Não & 134 & 31,60 & 104 & 25,37 & 1 & \\
\hline Sim & 290 & 68,40 & 306 & 74,63 & $1,17(0,99-1,38)$ & 0,05 \\
\hline \multicolumn{7}{|l|}{ Prematuridade } \\
\hline Não & 186 & 44,71 & 102 & 25,19 & 1 & \\
\hline Sim & 230 & 55,29 & 303 & 74,81 & $1,60(1,35-1,90)$ & $<0,01$ \\
\hline \multicolumn{7}{|l|}{ Baixo Peso } \\
\hline Não & 208 & 49,29 & 158 & 38,25 & 1 & \\
\hline Sim & 214 & 50,71 & 254 & 61,65 & $1,25(1,09-1,45)$ & $<0,01$ \\
\hline \multicolumn{7}{|l|}{ Apgar 10 minuto } \\
\hline Adequado & 283 & 71,28 & 276 & 69,17 & 1 & \\
\hline Baixo & 114 & 28,72 & 123 & 30,83 & $1,05(0,91-1,22)$ & 0,51 \\
\hline \multicolumn{7}{|l|}{ Apgar 5o minuto } \\
\hline Adequado & 364 & 91,69 & 376 & 94,24 & 1 & \\
\hline Baixo & 33 & 8,31 & 23 & 5,76 & $0,81(0,59-1,15)$ & 0,19 \\
\hline \multicolumn{7}{|c|}{$\begin{array}{l}\text { Uso de esteroide } \\
\text { antenatal }\end{array}$} \\
\hline Não & 275 & 86,48 & 236 & 76,13 & 1 & \\
\hline Sim & 43 & 13,52 & 74 & 23,87 & $1,36(1,15-1,61)$ & $<0,01$ \\
\hline
\end{tabular}

Fonte: elaborada pelas autoras com base nos dados da pesquisa. Nota: *Percentual na coluna.

Entre as intervenções clínicas realizadas nos neonatos, observou-se que a prevalência de suporte ventilatório com HOOD, uso do CPAP e dieta parenteral foi $91 \%, 89 \%$ e $18 \%$ maior entre os diagnosticados com desconforto respiratório $(p<0,01)$, nessa ordem. Por sua vez, a prevalência do uso de fórmulas e leite materno em neonatos com DRRN foi $85 \%$ e $62 \%$ menor $(p \leq 0,01)$ do que os neonatos que não apresentavam tal condição, respectivamente. As demais variáveis relativas às intervenções clínicas não apresentaram diferença estatisticamente significante (Tabela 3). 
Tabela 3 - Intervenções clínicas associadas ao diagnóstico de desconforto respiratório do recém-nascido. Cuiabá, Mato Grosso, Brasil, 2019.

\begin{tabular}{|c|c|c|c|c|c|c|}
\hline \multirow{3}{*}{ Variáveis } & \multicolumn{4}{|c|}{ Desconforto respiratório do recém-nascido } & \multicolumn{2}{|c|}{ Regressão de Poisson simples } \\
\hline & \multicolumn{2}{|c|}{ Não } & \multicolumn{2}{|c|}{ Sim } & \multirow{2}{*}{$\operatorname{RP}\left(\mathrm{IC}_{95 \%)}\right.$} & \multirow{2}{*}{ p-valor } \\
\hline & $\mathbf{n}$ & $\% *$ & $\mathbf{n}$ & $\% *$ & & \\
\hline \multicolumn{7}{|l|}{ Reanimação } \\
\hline Não & 261 & 49,53 & 266 & 50,47 & 1 & \\
\hline Sim & 89 & 49,72 & 90 & 50,28 & $0,99(0,77-1,28)$ & 0,96 \\
\hline \multicolumn{7}{|l|}{ Uso de surfactante } \\
\hline Não & 312 & 51,15 & 298 & 48,85 & 1 & \\
\hline Sim & 44 & 43,14 & 58 & 56,86 & $1,31(0,91-1,89)$ & 0,14 \\
\hline \multicolumn{7}{|c|}{ Suporte respiratório com HOOD } \\
\hline Não & 247 & 57,58 & 92 & 27,14 & 1 & \\
\hline Sim & 167 & 34,72 & 314 & 65,28 & $1,91(1,68-2,18)$ & $<0,01$ \\
\hline \multicolumn{7}{|c|}{ Suporte respiratório com CPAP } \\
\hline Não & 319 & 57,58 & 235 & 42,42 & 1 & \\
\hline Sim & 85 & 32,27 & 156 & 64,74 & $1,89(1,51-2,37)$ & $<0,01$ \\
\hline \multicolumn{7}{|l|}{ Ventilação mecânica } \\
\hline Não & 272 & 50,18 & 270 & 49,82 & 1 & \\
\hline Sim & 141 & 53,82 & 121 & 46,18 & $0,91(0,74-1,11)$ & 0,33 \\
\hline \multicolumn{7}{|l|}{ Leite Materno } \\
\hline Não & 335 & 47,25 & 374 & 52,75 & 1 & \\
\hline Sim & 78 & 72,90 & 29 & 27,10 & $0,38(0,25-0,57)$ & $<0,01$ \\
\hline \multicolumn{7}{|l|}{ LHP** } \\
\hline Não & 297 & 52,47 & 269 & 47,53 & 1 & \\
\hline Sim & 116 & 46,40 & 134 & 53,60 & $1,18(096-1,45)$ & 1,60 \\
\hline \multicolumn{7}{|l|}{ Fórmula } \\
\hline Não & 400 & 49,94 & 401 & 50,06 & 1 & \\
\hline Sim & 13 & 86,67 & 2 & 13,33 & $0,15(0,35-0,69)$ & $<0,01$ \\
\hline \multicolumn{7}{|l|}{ Dieta parenteral } \\
\hline Não & 207 & 55,65 & 165 & 44,35 & 1 & \\
\hline Sim & 206 & 46,40 & 238 & 53,60 & $1,18(1,04-1,34)$ & $<0,01$ \\
\hline
\end{tabular}

Fonte: elaborada pelas autoras com base nos dados da pesquisa.

Nota: *Percentual na linha ** Leite humano pasteurizado.

\section{DISCUSSÃO}

Os resultados do estudo apontaram que, entre os neonatos internados na UTIN estudada, 49,05\% foram diagnosticados com DRRN, sendo que a prevalência dessa morbidade foi maior entre os neonatos pré-termos, com baixo peso ao nascer e com histórico de uso materno de esteroide antenatal.

A relação encontrada entre o DRRN com a prematuridade e o baixo peso ao nascer, assemelha-se com outras pesquisas, as quais justificam que essa associação é decorrente da imaturidade pulmonar, sobretudo, nascimento antes do tempo e à fragilidade do neonato, ao realizar os esforços respiratórios ${ }^{(7-9)}$.

Embora não identificado neste estudo, a literatura revela associação do DRRN com o parto cesáreo em razão da ausência de compressão mecânica que o canal do parto desencadeia no tórax do neonato, auxiliando na expulsão do líquido pulmonar intrauterino(2-5). Outros fatores discutidos na literatura são o Apgar baixo, por sua ligação com a função respiratória, e o sexo masculino, o qual está associado ao amadurecimento tardio do pulmão ${ }^{(8-9)}$.

O uso materno do corticoide antenatal é altamente recomendado e encorajado para prevenir as complicações do parto prematuro. Segundo a diretriz do consenso europeu sobre o manejo da síndrome do desconforto respiratório, o uso desse medicamento pela mãe, antes ou durante 0 trabalho de parto, diminui comprovadamente a prevalência da doença, além de outras morbidades, como a hemorragia intraventricular e enterocolite necrosante ${ }^{(10)}$. Outro benefício de sua utilização é a redução de custos da assistência de saúde relacionada à diminuição da necessidade de um suporte neonatal especializado ${ }^{(11)}$.

Nesta pesquisa, apenas 43 mães (13,52\%) fizeram uso do corticoide antenatal, no entanto, identificou-se que a prevalência de DRRN foi maior entre os neonatos de mães que o utilizaram. Sabese que apenas a utilização do corticoide pela mãe, isoladamente, não garante a prevenção do DRRN, além disso, se administrado, tardiamente, pode ter sua ação prejudicada ${ }^{(12)}$. Ressalta-se que a 
pesquisa não investigou a idade gestacional em que foi administrado o esteroide, já que essa informação não constava nos registos dos prontuários. Sendo assim, outras intervenções terapêuticas combinadas podem garantir resultados mais satisfatórios.

O tratamento do DRRN consiste no recrutamento pulmonar, aplicando-se pressão positiva nas vias aéreas, por meio de ventilação não invasiva ou invasiva, associado ou não ao uso de surfactante exógeno ${ }^{(13)}$. Na presente pesquisa, a prevalência de suporte ventilatório com HOOD e CPAP foi maior entre os RN diagnosticados com DRRN, conforme esperado. A literatura evidencia a eficiência da ventilação não invasiva em $\mathrm{RN}$ e a tendência, cada vez maior, de seu uso em unidades neonatais $^{(14)}$. Pesquisas mostram que $o$ CPAP diminui a necessidade de traqueostomia e ventilação mecânica, além de estar associado à diminuição de displasia broncopulmonar e mortalidade neonatal ${ }^{(15-16)}$. Apesar do consenso sobre os benefícios da utilização da pressão positiva em vias aéreas em recém-nascidos, o seu uso excessivo e indevido acarreta efeitos adversos, como aumento da resistência vascular pulmonar, redução da perfusão pulmonar, diminuição do retorno venoso e baixo débito cardíaco ${ }^{(17)}$. Por isso, os profissionais de saúde, em especial a equipe de enfermagem, precisam estar atentos, para garantir o sucesso prático desse procedimento.

Nesse contexto, destaca-se a importância dos cuidados de enfermagem, para a prevenção das complicações da oxigenioterapia, avaliando e determinando as necessidades do neonato para uma prática segura, humanizada e pautada em evidências científicas, com o objetivo de promover a oxigenação adequada dos tecidos. Sabe-se que, durante $\mathrm{o}$ atendimento clínico de rotina, a resposta mais comum da equipe para episódios de hipoxemia é aumentar a concentração de oxigênio inspirado. Todavia, a intervenção correta exige que a enfermeira avalie esses pacientes e realize as medidas mais eficazes com base na fisiologia. Essas medidas podem incluir alterações nos parâmetros do ventilador, a aspiração das vias aéreas, reposicionamento da criança, dentre outros cuidados. Para tal, exigem-se profissionais de enfermagem preparados para intervir com rapidez e eficiência nas intercorrências, com o intuito de prevenir prováveis complicações e prestar uma assistência adequada ao recémnascido ${ }^{(2)}$.
O surfactante aparece no cenário de cuidados intensivos neonatais como medida de prevenção às complicações respiratórias. Estudos indicam que seu uso é eficaz para a melhora da função respiratória quando administrado em RN considerados de risco, como os pré-termos e os de baixo peso, e quando indicado de maneira precoce, até 2 horas após o nascimento. Além disso, a nova prática de administração menos invasiva do surfactante vem sendo difundida em todo o mundo e apresenta resultados positivos em relação ao uso invasivo ${ }^{(18)}$.

$\mathrm{Na}$ presente pesquisa, não houve associação significativa entre o DRRN e o uso de surfactante. Entretanto, estudos mostram que o CPAP, usado como forma de estabilização, na sala de parto, associado à administração precoce do surfactante ( $>2$ horas de vida) demonstra elevada eficácia, aumentando os resultados positivos do tratamento ${ }^{(15-16)}$.

Quanto ao suporte nutricional, observou-se que os neonatos com desconforto respiratório usaram menos fórmulas e leite materno. Aqui, cabe ressaltar que a maioria da população que apresentou DRRN era pré-termo e que, portanto, tem a introdução da dieta retardada, a depender da gravidade do seu quadro clínico e da idade gestacional. Além disso, a nutrição desses RN se inicia com a dieta parenteral e, depois, com o leite materno, na maioria das vezes pasteurizado, o que justificaria esse achado.

O padrão ouro da nutrição neonatal é o leite materno que auxilia no desenvolvimento, na prevenção de infecção, na microbiota, no alívio da dor, além de conter inositol e ômega 3 e 6 que estão ligados à prevenção da displasia pulmonar e ajudam a diminuir os efeitos da oxidação(19). Entretanto, em decorrência de fatores multifatoriais, em determinadas circunstâncias, não se pode estabelecer precocemente o aleitamento materno. Nessas situações, o LHP é a melhor opção, pois promove a formação da microbiota semelhante ao leite materno in natura e, em longo prazo, traz benefícios quanto ao funcionamento intestinal, fortifica o sistema imunológico e auxilia nos processos metabólicos (20).

Também existem casos em que o neonato apresenta intolerância à dieta enteral, principalmente, entre os pré-termos, nos quais o sistema cardiorrespiratório se encontra comprometido, em razão da distensão abdominal. Nessas circunstâncias, a dieta parenteral é 
fundamental para que o RN atinja o crescimento pós-natal adequado(21).

Considerando que os RNPT possuem maiores riscos para o desenvolvimento de distúrbios respiratórios que aqueles nascidos a termo, em decorrência da imaturidade pulmonar, os resultados deste estudo reafirmam a importância dos avanços na assistência à gestante de alto risco e no tratamento do neonato para a redução da morbimortalidade neonatal, principalmente pelo uso de substâncias exógenas, como a surfactante, o CPAP e a ventilação mecânica, dentre outras medidas terapêuticas, que têm desempenhado papel importante no aumento das taxas de sobrevida dos RN com deficiência respiratória ${ }^{(12,22-23)}$.

Quanto às limitações do estudo, sabe-se que a revisão de prontuários depende da qualidade das informações neles contidas, a incompletude e ausência de dados afetam, substancialmente, a análise fidedigna dos dados estudados. Apesar da pesquisa ter sido realizada em um hospital universitário, o emprego do prontuário eletrônico, nessa UTIN, ainda é recente e não abrangeu os prontuários analisados. Apesar disso, os resultados aqui apresentados retratam o contexto assistencial dessa temática e podem contribuir para melhoria da prática clínica e desenvolvimento de novas pesquisas.

\section{CONCLUSÃO}

Os resultados evidenciaram que metade dos neonatos internados, na UTIN, foi diagnosticada com DRRN, e os principais fatores associados ao evento foram a prematuridade e o baixo peso ao nascer.

Considerando que esses fatores demonstraram interferir diretamente nessa morbidade, indica-se maior atenção da equipe de saúde e gestores a esse perfil, pois tal conhecimento pode auxiliá-los no planejamento de ações para consolidação da rede perinatal, com reestruturação e qualificação dos processos assistenciais no pré-natal da gestante de alto risco, parto e no cuidado ao RN, além da capacitação de recursos humanos no manejo desse agravo neonatal.

Ademais, os resultados encontrados contribuem para a área da saúde e enfermagem, ao trazer à tona elementos que aprofundam o conhecimento sobre os DRRN e os principais fatores perinatais associados, auxiliando na melhor compreensão desse evento e das suas consequências para o neonato. Tais saberes são imprescindíveis para nortear ações e políticas de saúde com vistas ao planejamento de intervenções preventivas, além de contribuir para o avanço no campo teórico sobre o tema.

\section{REFERÊNCIAS}

1- Sweet LR, Keech C, Klein NP, Marshall HS, Tagbo BN, Quine D, et al. Respiratory distress in the neonate: Case definition \& guidelines for data collection, analysis, and presentation of maternal immunization safety data. Vaccine 2017;35(48):6506-17. DOI: 10.1016/j.vaccine.2017.01.046

2- Segur PC, Morero JAP, Oliveira CT. Assistência de Enfermagem ao recém-nascido com Síndrome do Desconforto Respiratório. Rev UNINGÁ 2019 [citado em 16 dez 2020]; 56(S2):141-59. Acesso em:

http://revista.uninga.br/index.php/uninga/article view/2071

3- Correia C, Rocha G, Flor-de-Lima F, Guimaraes $\mathrm{H}$. Respiratory morbidity in late preterm infants. Minerva Pediatr. 2018;70(4):345-54. DOI: 10.23736/s0026-4946.16.04580-1

4- Niesluchowska-Hoxha A, Cnota W, Czuba B, Ruci $A$, Ciaciura-Jarno $M$, Jagielska $A$, et al. retrospective study on the risk of respiratory distress syndrome in singleton pregnancies with preterm premature rupture of membranes between 24. Biomed Res Int. 2018:1-6. DOI: 10.1155/2018/7162478

5- Alfarwati TW, Alamri AA, Alshahrani MA, AlWassia $\mathrm{H}$. Incidence, risk factors and outcome of respiratory distress syndrome in term infants at Academic Centre, Jeddah, Saudi Arabia. Med Arch. 2019;73(3):183-6.

DOI: 10.5455/medarh.2019.73.183-186

6- França EB, Lansky S, Rego MAS, Malta DC, França JS, Teixeira $R$, et al. Principais causas da mortalidade na infância no Brasil, em 1990 e 2015: Estimativas do estudo de Carga Global de Doença. Rev Bras Epidemiol. 2017;20(1):46-60. DOI: 10.1590/1980-5497201700050005

7- Dias JPV, Costa MC, Sette DS, Nobre LN. Perfil clínico de neonatos internados em uma Unidade de Tratamento Intensivo Neonatal. Braz J Develop. 2019;5(10):22296-309. DOI: 10.34117/bjdv5n10$\underline{356}$

8- Donda K, Vijayakanthi N, Dapaah-Siakwan F, Bhatt P, Rastogi D, Rastogi S. Trends in epidemiology and outcomes of respiratory distress syndrome in the United States. Pediatr Pulmonol. 2019;54(4):405-14. DOI: 10.1002/ppul.24241 
9- Luo JH, Chen J, Li QP, Feng Z. Differences in Clinical Characteristics and Therapy of Neonatal Acute Respiratory Distress Syndrome (ARDS) and Respiratory Distress Syndrome (RDS): A retrospective analysis of 925 cases. Med Sci Monit. 2019;25:4992-8. DOI: 10.12659/MSM.915213

10- Sweet DG, Carnielli V, Greisen G, Hallman M, Ozek E, Pas A, et al. European Consensus Guidelines on the Management of Respiratory Distress Syndrome - 2019. Neonatol. 2019;115(4):432-50. DOI: 10.1159/000499361

11- Gyamfi-Bannerman C, Zupancic JA, SandovaL G, Grobman WA, Blackwell SC, Tita ATN, et al. Cost-effectiveness of antenatal corticosteroid therapy vs no therapy in women at risk of late preterm delivery: A secondary analysis of a randomized clinical trial. JAMA Pediatr. 2019;173(5):462-8. DOI:

\subsection{1/jamapediatrics.2019.0032}

12- Groom KM. Antenatal corticosteroids after 34 weeks' gestation: Do we have the evidence? Semin Fetal Neonatal Med. 2019;24(3):189-96. DOI: 10.1016/j.siny.2019.03.001

13- Fiorenzano DM, Leal GN, Sawamura KSS, Lianza AC, Carvalho WB, Krebs VLJ. Síndrome do desconforto respiratório: Influência do manejo sobre o estado hemodinâmico de recém-nascidos pré-termo $\leq 32$ semanas nas primeiras 24 horas de vida. Rev Bras Ter Intensiva 2019;31(3):312-7. DOI: $10.5935 / 0103-507 \times .20190056$

14- Ministério da Saúde (BR). Novas tendências em neonatologia. Portal de Boas Práticas em Saúde da Mulher, da Criança e dos Adolescentes 2019 [citado em 10 jun 2020]. Acesso em: https://portaldeboaspraticas.iff.fiocruz.br/atenca o-recem-nascido/novas-tendencias-emneonatologia/

15- Minocchieri S, Berry CA, Pillow JJ. Nebulised surfactant to reduce severity of respiratory distress: A blinded, parallel, randomised controlled trial. Arch Dis Child Fetal Neonatal Ed. 2019;104(3):313-9. DOI: 10.1136/archdischild2018-315051

16- Behnke J, Lemyre B, Czernik C, Zimmer KP, Ehrhardt $\mathrm{H}$, Waitz $\mathrm{M}$. Non-invasive ventilation in neonatology. Dtsch Arztebl Int. 2019;116(11):17783. DOI: $10.3238 /$ arztebl.2019.0177

17- Lakshminrusimha, S. The pulmonary circulation in neonatal respiratory failure. Clin Perinatol. 2012;39(3):655-83. DOI: 10.1016/i.clp.2012.06.006

18- Bae CW, Kim CY, Chung SH, Choi YS. History of pulmonary surfactant replacement therapy for neonatal respiratory distress syndrome in Korea. J
Korean Med Sci. 2019;34(25):e175. DOI: 10.3346/jkms.2019.34.e175

19- Ministério da Saúde (BR). Aleitamento materno na unidade neonatal. Portal de Boas Práticas em Saúde da Mulher, da Criança e dos Adolescentes 2018 [citado em 10 jun 2020]. Acesso

em:

https://portaldeboaspraticas.iff.fiocruz.br/atenca o-recem-nascido/aleitamento-materno-naunidade-neonatal/

20- Parra-Llorca A, Gormaz M, Alcántara C, Cernada $M$, Nuñez-Ramiro $A$, Vento $M$, et al. Preterm gut microbiome depending on feeding type: Significance of donor human milk. Front Microbiol. 2018;9:1376. DOI: 10.3389/fmicb.2018.01376

21- Davis JM, Pursley DM. Preventing long-term respiratory morbidity in preterm neonates: Is there a path forward? Pediatr Res. 2020;87(1):910. DOI: $10.1038 / \mathrm{s} 41390-019-0641-z$

22- Liu L, Oza S, Hogan D, Chu Y, Perin J, Zhu J, et al. Global, regional, and national causes of under5 mortality in 2000-15: An updated systematic analysis with implications for the Sustainable Development Goals. Lancet 2016;388(10063):3027-35. DOI: 10.1016/S01406736(16)31593-8

23- Teixeira JAM, Araujo WRM, Maranhão AGK, Cortez-Escalante JJ, Rezende LFM, Matijasevich A. Mortalidade no primeiro dia de vida: Tendências, causas de óbito e evitabilidade em oito Unidades da Federação brasileira, entre 2010 e 2015. Epidemiol Serv Saude 2019;28(1). DOI: $\underline{10.5123 / \text { S1679-49742019000100006 }}$

Nota: Este estudo é proveniente de um Trabalho de Conclusão de Curso de graduação.

Apoio financeiro da Coordenação de Aperfeiçoamento de Pessoal de Nível Superior (CAPES) por meio do Programa de Apoio à Pós-graduação (PROAP) da Universidade Federal de Mato Grosso.

Recebido em: 26/08/2020

Aprovado em: 17/12/2020

Endereço de correspondência:

Fabiane Blanco Silva Bernardino

Av. Fernando Correa da Costa, n. 2367

Bairro Boa Esperança, Cuiabá-MT

CEP:78060-900

fabianeblanco25@gmail.com 